\title{
Noble Metal-based Nanoparticles in Modern Bioanalysis
}

\author{
Warsaw University of Technology, Faculty of Chemistry, Chair of Medical Biotechnology, \\ Noakowskiego 3, 00-664 Warsaw, Poland \\ mariusz@ch.pw.edu.pl
}

\begin{abstract}
This talk will be focused on the synthesis and studies on various types of noble metal-based nanoparticles, which characterize themselves with catalytic activity mimicking enzymes from the class of oxidoreductases. Both the monometallic nanostructures as well as alloyed ones will be compared in terms of peroxidase-, catalase-, glucose oxidase- and superoxide dismutase-like activity. Moreover, core/shell and hollow nanoparticles, in some cases containing residues of templates inside, will be presented and their usefulness in various analytical applications discussed. It should be pointed out, that the latter ones are especially valuable, as they combine the capability for homogenous and heterogenous catalysis. Besides the analytical usefulness, some therapeutic capabilities of some of developed nanoparticles will be also indicated.
\end{abstract}

Key words: nanozymes, catalytic activity, nanoparticles, labels, therapy

\section{Introduction}

Due to the limitations such as: (i) high cost of isolation and (ii) covalent conjugation of enzymes with bioreceptors, together with their (iii) susceptibility to inactivation, the intensification of scientific efforts focused on replacement of enzymes, especially from the class of oxidoreductases, with their inorganic mimetics has been observed in recent years [1]. Various nanoparticles such as noble metalbased ones, graphene as well as other carbonbased nanomaterials and some metal oxides exhibit interesting properties, which allow to use them instead of enzymes for some applications. Like enzymes they can be applied in a role of receptor layer of sensors or as labels conjugated to proteins or nucleic acids, thus allowing the determination of many important analytes [2]. However, the common disadvantage of this type of catalyst, which is especially difficult to overcome, is the dependence of its activity on the state of catalyst surface. It should be pointed out, that the golden mean between ensuring both the adequate stability of nanoparticles (typically obtained by the surface-ligand interaction) and accessibility of their surface for substrate molecules must be found out. One of the class of stabilizers, which meets these requirements are hyperbranched polymers e.g. polyglycidol (see Fig. 1.) [3]. The interesting approach in this context seems to be the utilization of catalytic mechanism based on homogeneous catalysis, in which the activity is not derived from superficial atoms of high energy present on the surface of nanoparticles but intrinsic redox properties of released ions (e.g. from the nanoshells, see Fig. 2.).

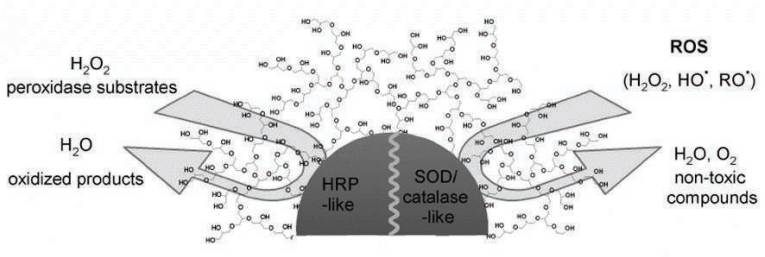

Fig. 1. Scheme of hyperbranched polyglycidolstabilized nanoparticle as peroxidase mimetic and ROS scavenger.

\section{Conducted studies}

In the framework of this project a number of nanoparticles, which differed by composition, structure and type of surface stabilizer (mostly hyperbranched poliglicydol and its derivatives) were synthesized and tested as mimetics of various oxidoreductases. The morphology of synthesized nanoparticles (see Fig. 3.), the dependence of their activity on applied conditions were tested and chosen nanostructures utilized for construction of biosensors. Moreover, a potential of developed 

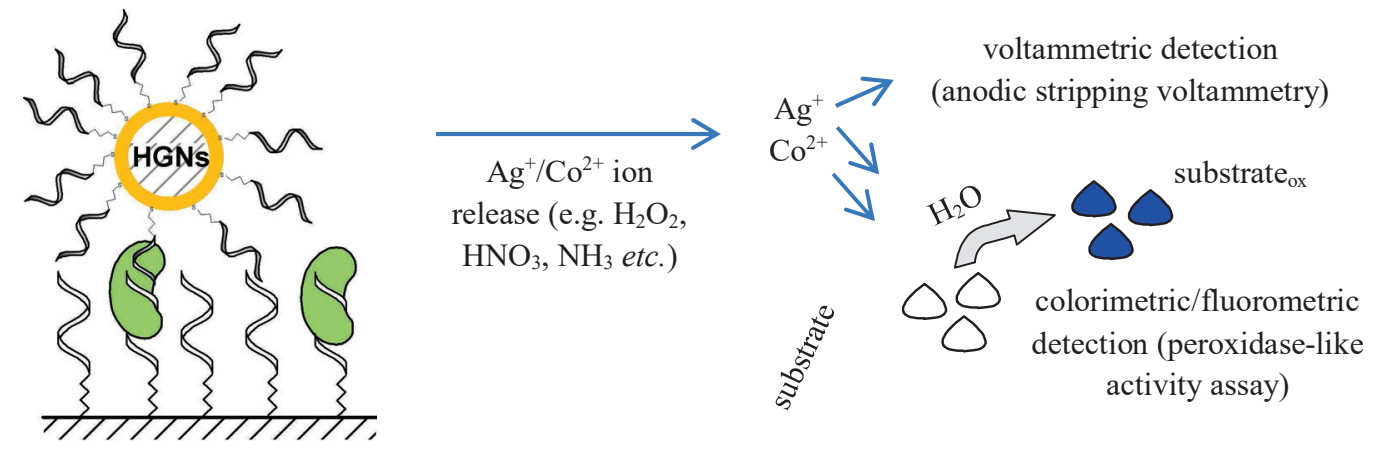

Fig.2. Schematic representation of the use of HGNs as electroactive/catalytic labels.

nanoparticles as scavengers of reactive oxygen species (ROS) and their cytotoxicity using various human cell lines to preliminarily verify, if it is safe to use them under in vivo conditions, were assessed.
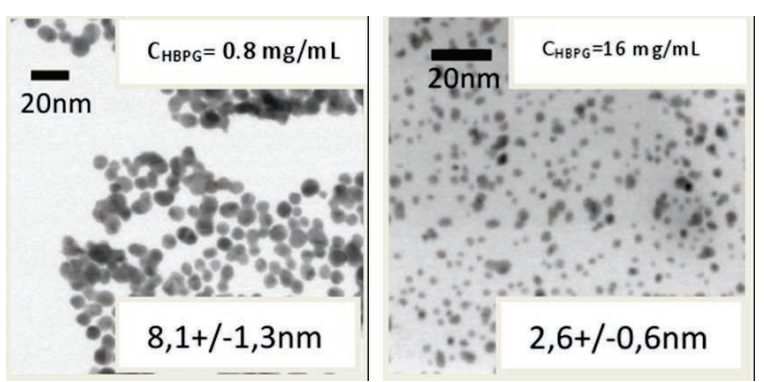

Fig. 3. Examples of TEM micrographs of AuNPs@HBPG (hyperbranched polyglycidol) prepared using solutions containing 0.8 or 16.0 $\mathrm{mg} / \mathrm{mL}$ of HBPG.

It should be underlined, that despite many differences of properties and mechanism of activity, in vast majority of the literature reports, characterization methods of catalytic properties of NPs were adapted directly from the studies related to native enzymes. Being aware of this fact, to reliably evaluate the catalytic activity of developed nanozymes, novel methods, not necessarily applicable for native enzymes, were proposed $[4,5]$.

\section{Acknowledgments}

This work was financially supported by the Polish National Science Centre - (NCN) grant 2017/01/X/ST5/00034 and Warsaw University of Technology.

\section{References}

[1] Y. Zhou, B. Liu, R. Yang, J. Liu, Filling in the Gaps between Nanozymes and Enzymes: Challenges and Opportunities, Bioconjugate chemistry 28, 2903-2909 (2017); doi: 10.1021/acs.bioconjchem.7b00673

[2] S. R. Ahmed, J. C. Corredor, É. Nagy, S. Neethirajan, Amplified visual immunosensor integrated with nanozyme for ultrasensitive detection of avian influenza virus, Nanotheranostics 1, 338-345 (2017); doi: 10.7150/ntno.20758

[3] M. Drozd, M. Pietrzak, P. Parzuchowski, M. Mazurkiewicz-Pawlicka, E. Malinowska, Peroxidase-like activity of gold nanoparticles stabilized by hyperbranched polyglycidol derivatives over a wide $\mathrm{pH}$ range, Nanotechnology 26, 495101 (2015); doi: 10.1088/0957-4484/26/49/495101

[4] M. Drozd, M. Pietrzak, J. Pytlos, E. Malinowska, Revisiting catechol derivatives as robust chromogenic hydrogen donors working in alkaline media for peroxidase mimetics, Analytica Chimica Acta 948, 80-89 (2016); doi: 10.1016/j.aca.2016.10.027

[5] M. Drozd, M. Pietrzak, P. Parzuchowski, E. Malinowska, Pitfalls and capabilities of various hydrogen donors in evaluation of peroxidase-like activity of gold nanoparticles, Analytical and Bioanalytical Chemistry 408, 8505-8513 (2016); doi: 10.1007/s00216-016-9976-Z 